\title{
Observation of an All-Solid-State Li-Ion Battery by In-Situ Electron Holography and Spatially-Resolved Electron Energy Loss Spectroscopy
}

\author{
Kazuo Yamamoto ${ }^{1}$, Takeshi Sato ${ }^{1}$, Ryuji Yoshida ${ }^{1}$, Hisanori Kurobe ${ }^{1}$, Hiroaki Matsumoto ${ }^{2}$, \\ Tsukasa Hirayama ${ }^{1}$, Yasutoshi Iriyama ${ }^{3}$ \\ ${ }^{1}$ Japan Fine Ceramics Center, 2-4-1 Mutsuno, Atsuta-ku, Nagoya, Aichi, 456-8587, Japan \\ ${ }^{2}$ Hitachi High-Technologies Corporation, 11-1 Ishikawacho, Hitachinaka, Ibaraki, 312-0057, Japan \\ 3 Department of Materials, Physics and Energy Engineering, Nagoya University, Furo-cho, \\ Chikusa-ku, Nagoya, Aichi, 464-8601, Japan
}

All-solid-state Li-ion batteries (LIBs) having incombustible solid electrolytes have been expected to be next generation energy-storage-devices with safety, high reliability and high energy density. However, a large interfacial resistance of Li-ion transfer at the electrode/solid-electrolyte interfaces prevents them from the practical use. We have so far developed technique to operate the LIB in a TEM and observed the electric potential change around the interfaces by electron holography (EH) $[1,2]$. However, it is still unclear how the Li-ions are distributed and what kind of relation $\mathrm{Li}$ concentration distribution and the potential profile have. In this report, we used spatially-resolved electron energy loss spectroscopy in a TEM mode (SR-TEM-EELS) to directly detect the Li concentration around the interface, and compared with the potential profile observed by EH.

A $90-\mu \mathrm{m}$ thick glass-ceramic sheet [3] $\left(\mathrm{Li}_{1+\mathrm{x}+\mathrm{y}} \mathrm{Al}_{\mathrm{y}} \mathrm{Ti}_{2-\mathrm{y}} \mathrm{Si}_{\mathrm{x}} \mathrm{P}_{3-\mathrm{x}} \mathrm{O}_{12}\right.$, LATSPO) was used as the solid electrolyte. The 800-nm thick $\mathrm{LiCoO}_{2}$ positive electrode was deposited on one side of the sheet by PLD. On the other side, the Pt-current-collector was directly deposited. Then, the cyclic voltammetry $(\mathrm{CV})$ was performed for 50 cycles in a vacuum with a sweep rate of $40 \mathrm{mV} / \mathrm{min}$. During the $\mathrm{CV}$, the Li-ions extracted from the $\mathrm{LiCoO}_{2}$ charge around the LATSPO/Pt interface, which decomposes the LATSPO, resulting in the formation of a negative electrode [4], as illustrated in Fig. 1(a). This in-situ formed negative electrode grows from the parent solid electrolyte by the $\mathrm{Li}$ insertion reaction, thus both the materials connect each other in an atomic scale, leading to a low interfacial resistance. The resistance was measured at most $100 \Omega \mathrm{cm}^{2}$, which was extremely lower than that at the $\mathrm{LiCoO}_{2}$-positive-electrode/LATSPO interface $\left(4000 \Omega \mathrm{cm}^{2}\right)$.

The negative side region surrounded by a broken line in Fig. 1(a) was selected by a rectangle slit in front of a spectrometer, and then the TEM image and spectrum image around Li-K-edge were recorded by a GIF CCD camera, as shown in Figs. 1(b) and 1(c). In the 400-nm region from the LATSPO/Pt interface, a slightly different contrast was observed in Fig. 1(b), where higher Li- ${ }^{-}$-edge $^{-}$ signals were detected in Fig. 1(c). Figure 1(d) shows the intensity profile of the Li signals along the 
A-B indicated in Fig. 1(c). The Li concentration distribution was clearly observed. Figure 2 shows the potential distribution observed by EH. Although the thickness of the negative electrode was different in places lifted out from the LIB bulk sample, both the distribution is very similar. In general, when the electrodes are electrochemically reduced by $\mathrm{Li}$ insertion, the electrode potential decreases. This is the consistent with the above experimental data. Therefore, EH can dominantly detect the changes of the electrode potential rather than that of the inner potential of Li.

In summary, we directly detected the Li concentration profile by SR-TEM-EELS, and showed that the potential profile obtained by EH corresponds to that of the Li concentration profile.

\section{References:}

[1] K. Yamamoto et al., Angew. Chem. Int. Ed. 49 (2010) 4414.

[2] K. Yamamoto et al., Electrochem. Commun.20 (2012) 113.

[3] J. Fu, J. Am. Ceram. Soc. 80 (1997) 1901.

[4] Y. Iriyama et al., Electrochem. Commun. 8 (2006) 1287.

[5] This work was supported by the RISING project of New Energy and Industrial Technology Development Organization (NEDO), Japan.

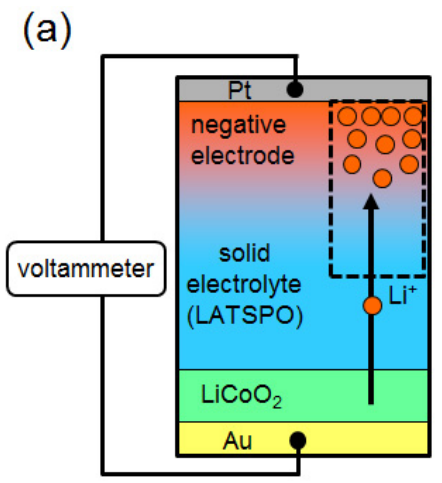

(b)

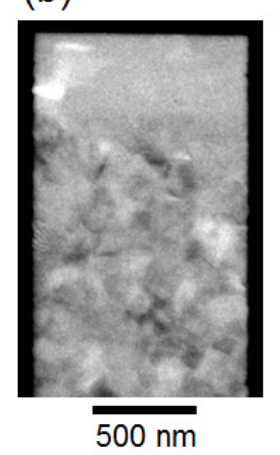

(c) $\mathrm{Li}$

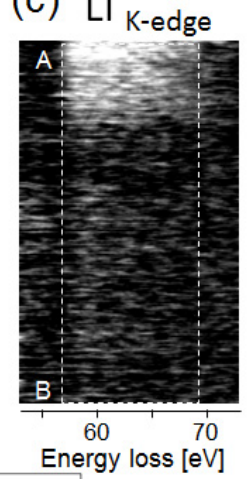

(d)

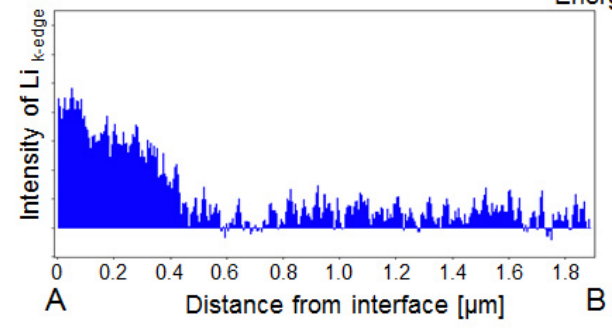

FIG. 1 Li distribution around the in-situ-formed-negative-electrode/solid-electrolyte interface detected by SR-TEM-EELS. (a) Schematic illustration of the LIB model sample, (b) TEM image around the interface, (c) spectrum image around $\mathrm{Li}_{\mathrm{k} \text {-edge, }}$ (d) $\mathrm{Li}$ distribution along the line $\mathrm{A}-\mathrm{B}$.

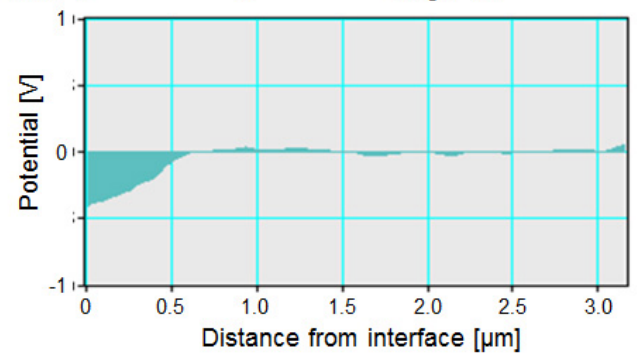

FIG. 2 Electric potential distribution observed by electron holography after initial cyclic voltammetry. 